\title{
Comparative study of efficacy and safety of intravenous ferric carboxy maltose versus iron sucrose in treatment of postpartum iron deficiency anemia
}

\author{
Roshni M. Patel, Mittal B. Bhabhor*, Nikita R. Pahwa, Simmy R. Ravani
}

Department of Obstetrics and Gynecology, AMCMET Medical College, Ahmedabad, Gujarat, India

Received: 19 December 2020

Accepted: 18 January 2021

\section{*Correspondence:}

Dr. Mittal B. Bhabhor,

E-mail: mittaldamor77@gmail.com

Copyright: ( $)$ the author(s), publisher and licensee Medip Academy. This is an open-access article distributed under the terms of the Creative Commons Attribution Non-Commercial License, which permits unrestricted non-commercial use, distribution, and reproduction in any medium, provided the original work is properly cited.

\begin{abstract}
Background: As postpartum iron deficiency anemia is observed in about $65 \%$ of women in India, it is the major contributing factor and indirect cause of maternal death. Postpartum anemia may lead to postpartum depression, stress, anxiety and cognitive impairment. Adequate treatment of anemia in postpartum period will have improved life quality in women in child bearing age so this study was designed with the objective to compare the safety and efficacy of intravenous FCM versus iron sucrose in treatment of postpartum iron deficiency anemia.

Methods: It was a prospective observational study of postpartum women (within 10 days of delivery) with iron deficiency anemia (7-9 gm\%) who delivered in LG hospital, Ahmedabad. A sample size of 215 women was estimated based on prevalence of anemia which is $65 \%$ among postpartum women in India including dropout rate of $10 \%$ which were further divided into 2 groups. Group 1: iron sucrose group, Group 2: ferric carboxy maltose group.

Results: FCM has greater rise in $\mathrm{Hb}$, less side effects, and easy administration of dose as compared to iron sucrose. The rise in $\mathrm{Hb}$ with FCM as compared to iron sucrose is (4.6 versus 3.5 respectively).

Conclusions: FCM has more safety and efficacy as compared to iron sucrose in treatment of postpartum iron deficiency anemia.
\end{abstract}

Keywords: Ferric carboxy maltose, Hemoglobin, Iron sucrose, Iron therapy, Postpartum anemia

\section{INTRODUCTION}

Postpartum iron deficiency anemia is a common problem in India. It is observed in about $65 \%$ of women. ${ }^{1}$ It is the major contributing factor and indirect cause of maternal death. Postpartum anemia may lead to postpartum depression, stress, anxiety and cognitive impairment. ${ }^{2,3}$ Adequate treatment of anemia in postpartum period will have improved life quality in women in child bearing age. ${ }^{4}$ Parenteral iron therapy is safe due to faster correction of $\mathrm{Hb}$ level with better compliance.

The objective of the study was to compare the safety and efficacy of intravenous FCM versus iron sucrose in treatment of postpartum iron deficiency anemia.

\section{METHODS}

It was a prospective observational study of postpartum women (within 10 days of delivery) with iron deficiency anemia (7-9 gm\%) who delivered in LG hospital. A sample size of 215 women was estimated based on prevalence of anemia which is $65 \%$ among postpartum women in India (Ministry of Health and Family Welfare. National Iron+ Initiative: Guidelines for Control of Iron Deficiency Anaemia, Adolescent Division, Ministry of Health and Family Welfare, Government of India, New Delhi; 2013) including dropout rate of $10 \%$.

The sample size of 215 women were divided into two groups: 1) Iron sucrose group $(200 \mathrm{mg} /$ day in $100 \mathrm{ml}$ $0.9 \%$ normal saline over 30 minutes on $0,2,4,6$ and 8 
day). 2) Ferric carboxy maltose (FCM) group (1000 mg in $100 \mathrm{ml} 0.9 \%$ normal saline over 30 minutes).

Both the formulations are free of cost as they are supplied by the hospital under the JSSK (Janani shishu suraksha karyakram) program for women and child development.

Diagnosis was confirmed by $\mathrm{CBC}$ and peripheral smear.

Follow up will be done after 6 weeks along with 1st day of her baby's immunization schedule on OPD basis for $\mathrm{CBC}$ investigation.

Any side effects (pain at injection site, itching and rash, abdominal pain, palpitation, headache, nausea, vomiting, anaphylactic shock) and compliance were be noted.

Written informed consent will be taken from all the patients enrolled in the study.

Calculation of iron requirement: $2.4 \times$ body weight (target $\mathrm{Hb}-$ actual $\mathrm{Hb}$ ) $+500 \mathrm{mg}$

\section{Inclusion criteria}

18-40 years old; postpartum (within 10 days of delivery) iron deficiency anemia (7-9 gm/dl); no history of significant vaginal bleeding in last 24 hours; no infection; intolerance to oral iron.

\section{Exclusion criteria}

History of parenteral iron intolerance; having thalassemia or sickle cell disease; history of bleeding disorders; non iron deficiency anemia; recent treatment with iron, blood transfusion or erythropoietin within 3 months; postpartum hemorrhage; previous blood transfusion; history of asthma or cardio vascular disease.

\section{RESULTS}

Out of the 215 patients treated for postpartum anemia 94 from iron sucrose group and 100 from FCM group completed the protocol. Those who completed protocol and came for follow up were included for study and statistical analysis.

Epidemiologically both groups were compared with age and parity.

Table 1: Distribution of subjects based on obstetric profile among two groups.

\begin{tabular}{|lllll|}
\hline Iron sucrose group & & \multicolumn{2}{c|}{$\begin{array}{l}\text { Ferric carboxy } \\
\text { maltose group }\end{array}$} \\
\hline Parity & n & \% & n & \% \\
\hline Multi gravida & 66 & 70.3 & 70 & 70 \\
\hline Primi gravida & 28 & 29.7 & 30 & 30 \\
\hline Total & 94 & 100 & 100 & 100 \\
\hline
\end{tabular}

Postpartum iron deficiency anemia was more common in multigravida patients than in primigravida (Table 1).

The most common age group affected with iron deficiency anemia was 18-25 years of women (Table 2).

Table 2: Age wise distribution of study subjects among two groups.

\begin{tabular}{|lllll|}
\hline \multirow{2}{*}{$\begin{array}{l}\text { Age } \\
\text { group }\end{array}$} & \multicolumn{2}{c}{ Iron sucrose group } & \multicolumn{2}{l|}{$\begin{array}{l}\text { Ferric carboxy } \\
\text { maltose group }\end{array}$} \\
& Frequency & Percent & Frequency & Percent \\
\hline $\mathbf{1 8 - 2 5}$ & 66 & 70.3 & 70 & 70 \\
\hline $\mathbf{2 6 - 3 0}$ & 18 & 19.2 & 22 & 22 \\
\hline $\mathbf{> 3 0}$ & 10 & 10.6 & 08 & 8 \\
\hline Total & 94 & 100 & 100 & 100 \\
\hline Mean & $31.33 \pm 4.41$ & & $33.33 \pm 4.62$ & \\
\hline
\end{tabular}

Sufficient rise in $\mathrm{Hb}$ (4.6 versus 3.5) was seen in patients receiving FCM as compared to iron sucrose (Table 3).

Table 3: Comparison of haemoglobin level among two groups.

\begin{tabular}{|lll|}
\hline Hb levels & $\begin{array}{l}\text { Iron sucrose } \\
\text { group } \\
\text { Mean } \pm \text { SD }\end{array}$ & $\begin{array}{l}\text { Ferric carboxy } \\
\text { maltose } \\
\text { Mean } \pm \text { SD }\end{array}$ \\
\hline Pre treatment & $7.79 \pm 0.69$ & $8.23 \pm 0.55$ \\
\hline Post treatment & $11.36 \pm 1.17$ & $12.84 \pm 1.04$ \\
\hline Hb difference & $3.5 \pm 1.12$ & $4.6 \pm 0.96$ \\
\hline
\end{tabular}

Less side effects (pain at injection site, itching and rash, abdominal pain, nausea, vomiting, palpitation, anaphylactic shock) were seen with administration of FCM (only 3 patients) as compared to iron sucrose (Figure 1).

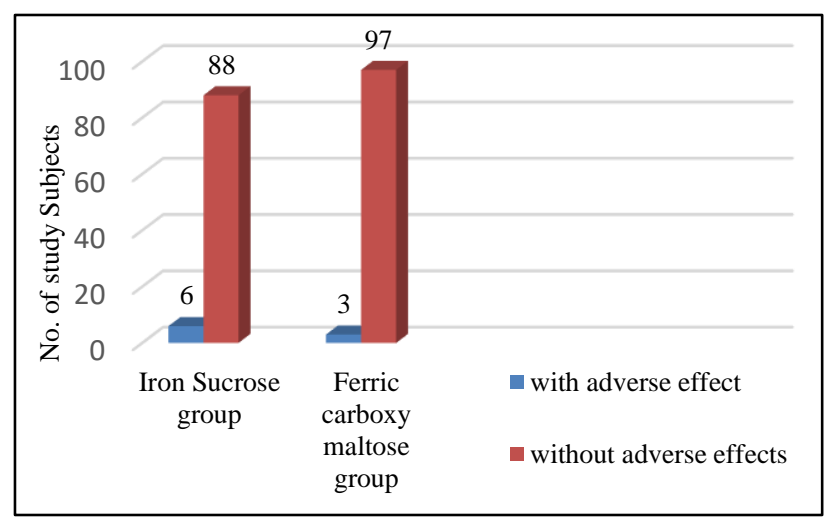

Figure 1: Distribution of the study subjects based on adverse effects among the two groups.

FCM is safer than ferric carboxy maltose as only $3 \%$ of study subjects of FCM group suffered from adverse effects as compared to $6.3 \%$ in iron sucrose group.

Since FCM is a single dose therapy as compared to iron sucrose ( 5 doses), hospital stay of patients receiving FCM is less as compared to patients receiving iron sucrose. 


\section{DISCUSSION}

Postpartum anemia is a major public health problem and is observed in $65 \%$ of women in India. Postpartum anemia arises frequently and imposes a substantial disease burden during the critical period of maternalinfant interactions. Anemic women have a longer average length of hospital stay, are more likely to receive a blood transfusion and incur higher hospitalization costs, Hence, postpartum IDA require attention and high quality care. ${ }^{5}$

Traditional treatments i.e. oral iron therapy and blood transfusion involves significant drawbacks. To overcome this problem IV iron preparations were used. ${ }^{6}$

Our study indicates that postpartum anemia can be treated effectively by FCM (ferric carboxy maltose) as compared to iron sucrose with additional advantage of single infusion, less side effects and better patient compliance.

In our study total 215 postpartum women with iron deficiency anemia were divided randomly in two groups, iron sucrose (group 1) and ferric carboxy maltose (group 2 ). Both groups were given deworming therapy, baseline investigations done, follow up was done at 6 weeks and $\mathrm{Hb}$ level repeated.

Singh et al in their study on 200 postpartum patients with Anemia and observed that there was mean rise of $\mathrm{Hb}$ was $2.086 \mathrm{gm}$ for FCM group and $1.766 \mathrm{gm}$ for iron sucrose group and Patel et al in their study showed the mean rise of hemoglobin value in Ferric carboxy maltose injection in the treatment of postpartum iron deficiency anemia was $5.2 \mathrm{gm} / \mathrm{dl}$ for ferric carboxy maltose and $4.1 \mathrm{gm} / \mathrm{dl}$ for iron sucrose in pregnant women. ${ }^{7,8}$

In our study mean rise of $\mathrm{Hb}$ in FCM group was 4.6 and in iron sucrose group was 3.5 which was significant.

Joshi et al in their study, most common age group affected was 18-25 years and Lunagariya et al study, most common age group was 20-25 years, in our study also most common age group is $18-25$ years. ${ }^{9,10}$

Joshi et al in their study, postpartum anemia was more common in multiparous women $(70-75 \%$ in both the groups). ${ }^{9}$ In our study also postpartum anemia was more common in multiparous women which accounts for nearly $70 \%$ in both groups.

In Joshi et al study, adverse effects were seen in $7.2 \%$ of iron sucrose group and $3.3 \%$ of FCM group whereas in our study $6.3 \%$ of patients of iron sucrose group and $3 \%$ of patients of FCM group developed side effects. ${ }^{9}$

Registered adverse effects were mild and quickly reversible and mostly restricted to local reaction at the infusion site. There was no treatment related serious adverse events. No anaphylactic reaction was detected.
No venous thrombosis was registered. Most common adverse effect in iron sucrose group was urticarial. ${ }^{11}$

Intra venous iron supplementation is highly effective in treating postpartum iron deficiency anemia, resulting in a much rapid resolution of iron deficiency anemia, has minimal side effects, and because it is administered intravenously, it circumvents problem of compliance. ${ }^{12}$

\section{CONCLUSION}

From our study we concluded that ferric carboxy maltose is safe and efficient in treatment of iron deficiency anemia in postpartum women as compared to iron sucrose, with lesser adverse effects and better patient compliance. Due to ultrashort duration of treatment i.e. ability to administer $1000 \mathrm{mg}$ dosage in a single sitting, fewer adverse effects, better compliance and decreased hospital stay in patients makes FCM the first line drug in management of postpartum iron deficiency anemia causing faster and higher replenishment of iron store and correction of $\mathrm{Hb}$ level. It increases patient compliance and decreases bed occupancy and burden on health facility in developing country like India.

\section{ACKNOWLEDGMENTS}

Authors would like to thank Dr. Yamini Trivedi, HOD, Department of obstetrics and Gynaecology for guidance in conducting the study efficiently and Dr. Sheetal Vyas, HOD, Department of Community Medicine for guidance in statistical analysis.

Funding: No funding sources

Conflict of interest: None declared

Ethical approval: Study was conducted after taking approval from institutional review board of LG hospital, Ahmedabad

\section{REFERENCES}

1. Ministry of Health and Family Welfare. National Iron+ Initiative: Guidelines for Control of Iron Deficiency Anaemia, Adolescent Division, Ministry of Health and Family Welfare, Government of India, New Delhi; 2013. Available from: https://www.nhm.gov.in/images/pdf/programmes/chi ld-health/guidelines/Control-of-Iron-Deficiency-

Anaemia.pdf. Accessed on 20 August 2020.

2. Bodnar LM, Cogswell ME, Mc Donald. Have we forgotten the significance of postpartum iron deficiency? Am J Obstet Gynecol. 2005;193(1):3644.

3. Bodnar LM, Scanlon KS, Freedman DS, Siega RAM, Cogswell ME. High prevalence of postpartum anemia among low-income women in the United States. Am J Obstet Gynecol. 2001;185(2):438-43.

4. Beard J, Hendricks M, Perez E, Murray-Kolb L, Berg A, Vernon-Fegans L. Maternal iron deficiency 
affects postpartum emotion and cognition. J Nutr. 2005;135:267-72.

5. Pfenniger A, Schuller C, Christoph P, Surbek D. Safety and efficacy of high-dose intravenous iron carboxymaltose vs. iron sucrose for treatment of postpartum anemia. $\mathrm{J}$ Perinat Med. 2012;40(4):397402.

6. Seid MH, Mangione A, Valaoras TG, Anthony LB, Barish CF. Safety profile of iron carboxymaltose, a new high dose intravenous iron in patients with iron deficiency anemia. Blood. 2006;108:3739.

7. Singh S, Dhama V, Chaudhary R, Singh P. Comparing the safety and efficacy of intravenous iron sucrose and intravenous ferric carboxymaltose in treating postpartum anemia. Int $\mathrm{J}$ Reprod Contracept Obstet Gynecol. 2016;5:1451-6.

8. Patel J, Patel K, Patel J, Sharma A, Date SK. Comparison of intravenous iron sucrose and ferric carboxymaltose therapy in iron deficiency anemia during pregnancy and postpartum period. J Pharm Sci Bioscientific Res. 2015;5:239-43.

9. Joshi SD, Chikkagowdra S, Kumar VC. Comparative study of efficacy and safety of intravenous ferric carboxy maltose versus iron sucrose in treatment of postpartum iron deficiency anemia. Int $\mathrm{J}$ Reprod Contracept Obstet Gynecol. 2016;5(8):2566-70.

10. Lunagariya M, Nakum KD, Aditi V, Patel J, Patel M. Iron Sucrose versus ferric carboxymaltose: in search of better treatment option in cases of post partum iron deficiency anemia. Int J Contemp Med Res. 2018;5(1).

11. Seid MH, Derman RJ, Baker JB, Banach W, Goldberg C, Rogers R. Ferric carboxymaltose injection in the treatment of postpartum iron deficiency anaemia: a randomized controlled clinical trial. Am J Obstet Gynecol. 2008;199:435:1-7.

12. Crichton R, Danielson B, Geisser P. Iron therapy. In: A Book. 1st ed. Bremen, Germany: Uni-Med Verlag; 2005.

Cite this article as: Patel RM, Bhabhor MB, Pahwa NR, Ravani SR. Comparative study of efficacy and safety of intravenous ferric carboxy maltose versus iron sucrose in treatment of postpartum iron deficiency anemia. Int J Reprod Contracept Obstet Gynecol 2021;10:707-10. 